\title{
MONET, HET and SALT and asteroseismological observations and theory in Göttingen
}

\author{
S. Schuh, F. V. Hessman, S. Dreizler, W. Kollatschny, W. Glatzel \\ Institut für Astrophysik, Georg-August-Universität Göttingen, 37077 Göttingen, Germany
}

\begin{abstract}
The Göttingen stellar astrophysics group, headed by Stefan Dreizler, conducts research on extrasolar planets and their host stars, on lower-main sequence stars, and on evolved compact objects, in particular hot white dwarfs (including PG 1159 objects, magnetic WDs and cataclysmic variables), and subdwarf B stars.

In addition to sophisticated NLTE spectral analyses of these stars, which draw on the extensive stellar atmosphere modelling experience of the group, we actively develop and apply a variety of photometric monitoring and time-resolved spectroscopic techniques to address time-dependent phenomena.

With the new instrumentational developments described below, we plan to continue the study of variable white dwarfs (GW Vir, DB and ZZ Ceti variables) and in particular sdB EC 14026 and PG 1617 pulsators which already constitute a main focus, partly within the Whole Earth Telescope (WET/DARC), http://www.physics.udel.edu/ jlp/darc/) collaboration, on a new level. Additional interest is directed towards strange mode instabilities in Wolf Rayet stars.
\end{abstract}

\section{MONET}

MONET (http://monet.uni-goettingen.de) is a MOnitoring NEtwork of Telescopes consisting of two $1.2 \mathrm{~m}$ internet-operated telescopes with $40 \%$ of observing time reserved for school use. To improve physics and computer instruction in participating international schools, the Alfried Krupp von Bohlen und Halbach-Stiftung has awarded funds for the construction of these fully automatic robotic telescopes, which are built by Teleskoptechnik Halfmann. Our partner institutions, which host and run the telescopes, are the McDonald Observatory of the University of Texas at Austin and the South African Astronomical Observatory with its station at Sutherland. The first telescope (MONET/North) was erected at McDonald in December 2005. Remote observing has been put into operation and is routinely done, so that several scientific projects are now running to evaluate the performance of this new facility. The delivery of MONET/South into its already finished enclosure at Sutherland is scheduled for later this year and it should be commissioned in early 2007. MONET/South should be able to serve as a pathfinder telescope for SALT.

The two MONET telescopes will not only represent a network by themselves: The HTN (http://www.telescope-networks.org) collaboration plans on joining a large variety of telescopes with different specific purposes in a Heterogeneous Telescope Network. It is also planned to use the MONET telescopes to participate in future WET/DARC (Whole Earth Telescope / Delaware Asteroseismic Research Center) campaigns. One of several primary goals of MONET during scientific use is the study of stellar variability.

\section{HET and SALT}

As a partner in these telescope projects, the Institute for Astrophysics Göttingen has guaranteed access to the HET (http://www.as.utexas.edu/mcdonald/het/het.html) and SALT 
(http://www.salt.ac.za). The planned high time resolution in combination with the projected UV capabilities make SALT, in particular, attractive for studies of compact blue variable objects.

\section{Asteroseismology projects}

In the past, our contributions to $\mathrm{sdB}$ monitoring were predominantly obtained with Calar Alto observatory telescopes (e.g. the discovery and follow-up monitoring of the hybrid sdB pulsator HS 0702+6043, and part of the extensive monitoring of PG 1605+072 during the MultiSite Spectroscopic Telescope (MSST) campaign, and long-term monitoring of HS 2201+2610).

As a special performance verification project for SALT, we currently are investigating the photometric stability of Wolf-Rayet stars with SALTICAM to test existing models that predict strange mode oscillations, and we obtain spectra with RSS to check classification and search for line profile variations.

Acknowledgments. S. Schuh thanks the organizers for generous support.

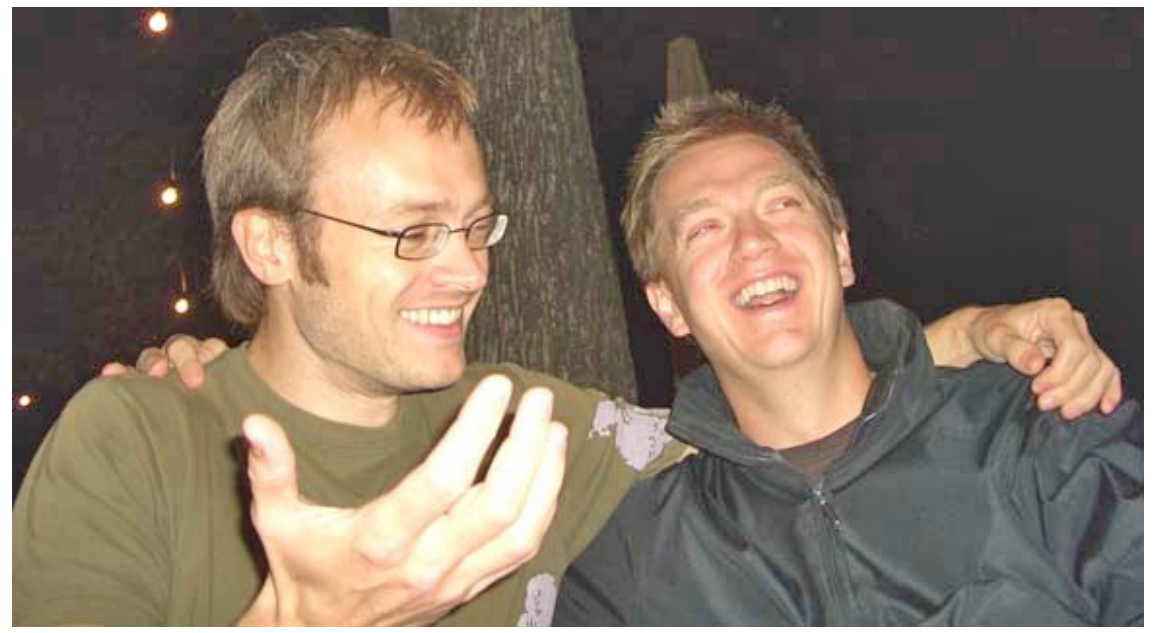

Hans Bruntt and Frank Grundahl enjoying a pleasantly warm Viennese evening. 